\title{
Impact of SARS-CoV-2 on Progression of Glycemic and Cardiometabolic Variables and Changes in Insulin Indices: A Longitudinal Study
}

\author{
Alpesh Goyal · Yashdeep Gupta (D) · Mani Kalaivani · Neerja Bhatla • \\ Nikhil Tandon
}

Received: August 17, 2021 / Accepted: September 17, 2021 / Published online: October 5, 2021

(C) The Author(s) 2021

\section{ABSTRACT}

Introduction: We aimed to evaluate whether SARS-CoV-2 infection is associated with beta cell dysfunction and progression of glycemic and cardiometabolic variables in an established cohort.

Methods: Study participants $(n=352,46.9 \%$ men) underwent a detailed evaluation at two time points: (a) pre-COVID (2016-19) and (b) peri-COVID (2020-21). At the second visit, SARS-CoV-2 infection was determined on the basis of a quantitative S1/S2 IgG antibody test (DiaSorin Liaison) and/or a documented history of infection.

Results: A total of 159 (45.2\%) participants were seropositive for SARS-CoV-2, of whom 122 (76.7\%) had mild/asymptomatic infection. Progression in body mass index (BMI) category [34 $(21.4 \%)$ vs. $22(11.4 \%), p=0.011]$ was seen

A. Goyal · Y. Gupta $(\bowtie) \cdot$ N. Tandon Department of Endocrinology and Metabolism, All India Institute of Medical Sciences, New Delhi 110029, India

e-mail: yash_deep_gupta@yahoo.co.in

M. Kalaivani

Department of Biostatistics, All India Institute of Medical Sciences, New Delhi 110029, India

N. Bhatla

Department of Obstetrics and Gynecology, All India Institute of Medical Sciences, New Delhi 110029, India in a significantly higher proportion of the participants in the infected group compared to the non-infected group. Progression in glycemic and insulin indices [homeostasis model assessment of insulin resistance (HOMA-IR), Matsuda index, and oral disposition index (oDI)] categories was also evident in a larger proportion of participants in the infected group; however, the difference was not statistically significant. On logistic regression analysis, the association between SARS-CoV-2 infection and BMI category progression was statistically significant [fully adjusted OR 2.14 (95\% CI, 1.18-3.90; $p=0.013)]$.

Conclusion: In this longitudinal study, predominant mild/asymptomatic SARS-CoV-2 infection was associated with increase in BMI, but not with worsening of beta cell function and insulin resistance, nor glycemic progression.

Keywords: SARS-CoV-2 infection; Beta cell dysfunction; COVID-19; Diabetes; Obesity 


\section{Key Summary Points}

\section{Why carry out this study?}

Diabetes and COVID-19 carry a bidirectional relationship. There is a paucity of data on the exact magnitude and pathophysiological link for beta cell dysfunction and new-onset diabetes following COVID-19, especially mild/ asymptomatic disease.

This study aimed to evaluate whether SARS-CoV-2 infection is associated with beta cell dysfunction and progression of glycemic and cardiometabolic variables in an established cohort.

\section{What was learned from the study?}

A significantly higher proportion of participants in the infected group progressed in body mass index (BMI) category, i.e., from normal weight to overweight or from overweight to obesity category.

Progression in glycemic and insulin indices [homeostasis model assessment of insulin resistance (HOMA-IR), Matsuda index, and oral disposition index (oDI)] categories was also evident in a larger proportion of participants in the infected group; however, the difference was not statistically significant.

To the best of our knowledge, this is the first study to formally evaluate the emerging concept of new-onset metabolic dysfunction following SARS-CoV-2 infection using a longitudinal cohort design.

\section{INTRODUCTION}

The ongoing coronavirus disease 2019 (COVID19) pandemic has badly affected the global health community. At the time of writing this paper, more than 187 million cases and more than 4.0 million deaths have already been reported [1]. Diabetes and COVID-19 share a bidirectional relationship. On the one hand, poor COVID-19 outcomes have been reported in patients with uncontrolled hyperglycemia [2-4]; on the other hand, the viral infection itself has been postulated to affect beta cell function, and induce new-onset diabetes and severe metabolic decompensation in patients with pre-existing diabetes [5-7]. Data on the exact magnitude and pathophysiological link for beta cell dysfunction and new-onset diabetes following COVID-19 are still very scarce. To address this lacuna, a global registry of patients with COVID-19-related diabetes has been established [8]. The registry includes patients with new-onset diabetes with confirmed severe acute respiratory syndrome coronavirus 2 (SARS-CoV-2) infection, a negative past history of diabetes, and a previously normal glycated hemoglobin (HbA1c) level. However, it remains to be seen whether the registry provides insights into exact mechanisms for virus-induced beta cell dysfunction and metabolic deterioration.

There are some salient aspects to the pathophysiology of diabetes epidemic in South Asian countries like India. Type 2 diabetes is known to develop at a younger age and at a lower body mass index (BMI) in Asian Indians, compared to white Caucasians [9]. Besides, compared to other ethnicities, beta cell secretory defect, rather than insulin resistance, is the more prominent pathophysiological defect [10]. Given the proposed link between SARS-CoV-2 infection and beta cell dysfunction, it would be interesting to examine the trends in beta cell function and glycemic parameters in a South Asian cohort evaluated before and during/after the course of pandemic.

With this background, we evaluated participants, aged 20-50 years, from a cohort of women with and without history of hyperglycemia in pregnancy and their spouses, which has been previously established. The participants in this cohort underwent a detailed evaluation for glycemic parameters and insulin indices at two points: (a) pre-COVID period (2016-19) and (b) peri-COVID period (2020-21). Besides, at the second visit, SARS- 
CoV-2 infection was determined on the basis of the presence of SARS-CoV-2 IgG antibodies and/ or a documented history of viral infection. The dual evaluation planned in this study provides us an opportunity to determine whether SARS$\mathrm{CoV}-2$ infection is associated with worsening of beta cell function, and insulin resistance and progression of glycemic and cardiometabolic parameters. To the best of our knowledge, this is the first study to formally evaluate the emerging concept of new-onset metabolic dysfunction following SARS-CoV-2 infection using a longitudinal cohort design.

\section{METHODS}

\section{Settings and Study Design}

This was a longitudinal cohort study performed at a public tertiary care center in North India (All India Institute of Medical Sciences, New Delhi) that caters to the low- and middle-income population. The cost for all study-related procedures and investigations was exempted for participants, as per the study protocol. The study was approved by the institutional ethics committee (IEC ref. no. IEC-110/05.02.2021) and informed consent was obtained from the study participants. The study was conducted in accordance with the International Conference on Harmonisation Guidelines for Good Clinical Practice and the Declaration of Helsinki.

\section{Inclusion and Exclusion Criteria}

Women with and without history of hyperglycemia in pregnancy and their spouses who are part of an existing cohort were included in this study. To be eligible, a participant should have been evaluated twice: (a) before the onset of COVID-19 outbreak (pre-COVID period; 2016-19), and (b) during the time of COVID-19 outbreak (peri-COVID period; 2020-21). Since the study aimed to evaluate the progression of glycemic parameters, subjects with diabetes at the initial visit were excluded.

\section{Study Procedures}

At both the study visits, participants were invited to attend the hospital in a fasting state (minimum fast of $10 \mathrm{~h}$ ). At the scheduled visit, participants underwent a 75-g oral glucose tolerance test with measurement of plasma glucose and serum insulin at 0,30 and $120 \mathrm{~min}$. Besides, blood was also collected in the fasting state for measurement of HbA1c. On both the study visits, anthropometric measurements were performed using standard methods. Details regarding the anthropometric and biochemical variables and their measurements, including the method of performing oral glucose tolerance test and calculation of insulin indices, such as homeostasis model assessment of insulin resistance (HOMA-IR), a marker of insulin resistance and oral disposition index (oDI), a marker of composite beta cell function, were provided in our previous publications $[11,12]$. Briefly, HOMA-IR was calculated using the following formula: fasting insulin $(\mathrm{mU} / \mathrm{L}) \times$ fasting plasma glucose $(\mathrm{mmol} / \mathrm{L}) / 22.5$; and ODI was calculated using the following formula: $\Delta \mathrm{I} 0-30 / \Delta \mathrm{G} 0-30 \times 1 /$ fasting insulin (where $\Delta \mathrm{IO}-30$ is the change in serum insulin over $30 \mathrm{~min}[\mathrm{pmol} / \mathrm{L}]$ and $\Delta \mathrm{GO}-30$ is the change in plasma glucose over $30 \mathrm{~min}$ [mmol/L]) [13-15]. An online web calculator was used to calculate the Matsuda index (a marker of insulin sensitivity) $[16,17]$.

A detailed questionnaire documenting demographic details, education and employment status, and family history of diabetes was filled at the initial visit. Additionally, during the second visit (peri-COVID period; 2020-21), blood was collected for SARS-CoV2 IgG antibody test, and a history of documented SARSCoV-2 infection [using reverse transcription polymerase chain reaction (RT-PCR) or rapid antigen test] was ascertained. Accordingly, the participants were classified into two groups: (a) infected (antibody positive, defined as levels $\geq 15 \mathrm{AU} / \mathrm{mL}$ and/or history of documented infection), and (b) non-infected (antibody negative, defined as levels $<12 \mathrm{AU} / \mathrm{mL}$ and no history of documented infection). Participants who tested antibody positive, but had no prior history of documented SARS-CoV-2 
infection, were classified as having mild/ asymptomatic infection. The last study sample was collected on 26 February 2021, about 3 days before the Government of India initiated the vaccination drive for the high-risk general population [18]. Thus, the seropositivity data obtained in this study were not affected by the ongoing vaccination program.

\section{Study Definitions}

Normoglycemia was defined as fasting plasma glucose of $<100 \mathrm{mg} / \mathrm{dL}(5.6 \mathrm{mmol} / \mathrm{L})$, and $2 \mathrm{~h}$ plasma glucose of $<140 \mathrm{mg} / \mathrm{dL}(7.8 \mathrm{mmol} / \mathrm{L})$. Prediabetes was defined as the presence of impaired fasting glucose [fasting plasma glucose of 5.6-6.9 mmol/L (100-125 mg/dL)], impaired glucose tolerance [ $2 \mathrm{~h}$ plasma glucose of $7.8-$ $11.0 \mathrm{mmol} / \mathrm{L}(140-199 \mathrm{mg} / \mathrm{dL})]$, or both. Diabetes mellitus was defined as fasting plasma glucose of $\geq 7.0 \mathrm{mmol} / \mathrm{L}(126 \mathrm{mg} / \mathrm{dL})$ and/or $2 \mathrm{~h}$ plasma glucose of $\geq 11.1 \mathrm{mmol} / \mathrm{L}(200 \mathrm{mg} /$ dL) and/or HbA1c of $\geq 48 \mathrm{mmol} / \mathrm{mol}(6.5 \%)$ [19]. Overweight and obesity were defined as BMI 25-29.9 and $\geq 30 \mathrm{~kg} / \mathrm{m}^{2}$, respectively [20]. Hypertension was defined as a blood pressure $\geq 140 / 90 \mathrm{mmHg}$ and/or use of antihypertensive medications [21].

Progression in glycemic category was defined as worsening from normoglycemia to prediabetes/diabetes or from prediabetes to diabetes over the course of two study visits. Progression in BMI category was defined as worsening from normal weight to overweight/obese state or from overweight to obese state. Progression in insulin indices, namely HOMA-IR, Matsuda index, and oDI, was defined when the value obtained on the follow-up visit crossed the original tertile category (e.g., from first to second or third tertile, and from second to third tertile).

\section{Biochemical and Hormonal Measurements}

Plasma glucose was measured on an autoanalyzer using the hexokinase method (Cobas Integra 400 Plus analyzer, Roche Diagnostics, Mannheim, Germany). HbA1c was measured using an ion-exchange high-performance liquid chromatography method (Tosoh HLC-723 G8 HbA1c analyzer, Tosoh Corp., Tokyo, Japan). The inter-assay coefficients of variation $(\mathrm{CV})$ for HbA1c derived from low- and high-quality control samples were $<3.0 \%$. Serum insulin was measured using an electrochemiluminescent tracer-based two-site immunometric assay (Cobas e-411 analyzer, Roche Diagnostics, Mannheim, Germany). The inter-assay CVs for serum insulin based on low- and high-quality control samples were $4.0 \%$ and $3.8 \%$, respectively. The departmental laboratories participate in external quality assurance programs for various study analytes, namely glucose and HbA1c (EQAS Bio-Rad) and insulin (UK NEQAS, Guildford).

\section{SARS-CoV-2 IgG Antibody Test}

IgG antibodies against S1 and S2 proteins of SARS-CoV-2 were detected using an indirect chemiluminescence immunoassay (CLIA) on the LIAISON ${ }^{\circledR}$ XL autoanalyser (DiaSorin S.p.A., Saluggia, Italy). The assay provides quantitative estimation of antibody concentration. As per the manufacturer's kit insert, antibody concentration $<12 \mathrm{AU} / \mathrm{mL}$ was considered as negative and $>15 \mathrm{AU} / \mathrm{mL}$ as positive. The diagnostic sensitivity and specificity for this antibody assay are $97.4 \%$ ( $>15$ days after confirmed microbiological diagnosis) and 98.5\%, respectively. In a previous study, antibody levels $>80 \mathrm{AU} / \mathrm{mL}$ using this assay platform were shown to have $87 \%$ positive agreement with plaque reduction neutralization test (PRNT) titers $>1: 160$ [22].

For samples with intermediate concentration between 12 and $15 \mathrm{AU} / \mathrm{mL}$, retesting of the same specimen in duplicate was performed, as per the manufacturer's instructions. Samples having at least 2 out of the 3 results equal to or above $15.0 \mathrm{AU} / \mathrm{mL}$ were graded as positive, while those with at least 2 out of the 3 results less than $12.0 \mathrm{AU} / \mathrm{mL}$ or a repeat indeterminate result were graded as negative. The limit of detection for the assay was $3.8 \mathrm{AU} / \mathrm{mL}$ and the measurement range was up to $400 \mathrm{AU} / \mathrm{mL}$. For samples with concentration less than limit of detection and more than measurement range, 
values of $3.8 \mathrm{AU} / \mathrm{mL}$ and $400 \mathrm{AU} / \mathrm{mL}$, respectively were entered. The intra- and inter-assay $\mathrm{CV}$ for the assay were $5.0 \%$ and $8.4 \%$, respectively.

\section{Sample Size Calculation}

There were no data on the proposed research question to guide sample size calculation at the time of drafting this study. Therefore, the sample size calculations were based on the following observations: (a) the COVID-19 positivity rate was assumed to be $30 \%$, based on seroprevalence estimates from New Delhi, India at that time [23], and (b) the deterioration in glycemic category (from normoglycemia to prediabetes/diabetes and from prediabetes to diabetes) was assumed to be $30 \%$ and $15 \%$, respectively, in participants with and without COVID-19. Using these assumptions, and with an $80 \%$ power and two-sided $\alpha=0.05$, the required sample size was determined to be 350 .

\section{Statistical Analysis}

STATA 15.0 (Stata Corp, College Station, TX, USA) was used for the statistical analyses. Data are presented as number (\%), mean \pm standard deviation or median (q25-q75), as appropriate. For comparison of qualitative variables between two groups, Pearson's $\chi^{2}$ test or Fisher's exact test were used, as appropriate. Normally distributed quantitative variables were compared using Student's $t$ test, while Wilcoxon rank sum test or Kruskal-Wallis test were used for comparing quantitative variables without normal distribution. Logistic regression analysis was used to evaluate association between SARS-CoV2 infection and progression of glycemic and BMI categories. The results of regression analysis were expressed as unadjusted and adjusted odds ratio (95\% confidence interval [CI]). For adjusted analysis, the following covariates that are known to have a bearing on the outcome were accounted: age, gender, employment status, and education (model 1); baseline BMI, and family history of diabetes (model 2); duration between two study evaluations (model 3); and all the aforementioned covariates combined (model 4). The significance level was set at $p<0.05$.

\section{RESULTS}

\section{Baseline Characteristics}

A total of 352 participants [165 (46.9\%) men] were evaluated in this study. The mean age at the time of initial visit was $35.2 \pm 5.3$ years. Family history of diabetes was present in 130 (36.9\%) participants. A total of 244 (69.3\%) participants were normoglycemic, while another 108 (30.7\%) had prediabetes. A total of 194 (55.1\%) participants were either overweight or obese. Hypertension was present in 34 (9.7\%) participants (Table 1).

\section{Seroprevalence and Infection Data}

A total of 159 (45.2\%) participants were seropositive for SARS-CoV-2 IgG. A history of documented infection was present in 37 (10.5\%) participants. Among these, 29 (78.4\%) tested positive for RT-PCR test, while rest 8 $(21.6 \%)$ tested positive on a rapid antigen test. All participants $(n=37)$ with a history of documented infection were seropositive, while a total of $122(76.7 \%)$ seropositive individuals had no history of SARS-CoV-2 infection, suggestive of asymptomatic or mild self-limited disease (Fig. 1). The median antibody levels in study participants $(n=352) \quad$ was 8.2 (3.8-51.5) AU/mL, while that in seropositive individuals $(n=159)$ was $63.4(33.9-106.0) \mathrm{AU} /$ $\mathrm{mL}$. Among seropositive individuals, 54 (34.0\%) had antibody levels between 15 and $40 \mathrm{AU} / \mathrm{mL}$, while $46(28.9 \%)$ and 59 (37.1\%) had antibody levels between 40 and $80 \mathrm{AU} / \mathrm{mL}$, and $>80 \mathrm{AU} /$ $\mathrm{mL}$, respectively. On subgroup analysis, seropositivity rate and antibody titers (among seropositive participants) were not statistically different ( $p=0.744$ and 0.839 , respectively) between participants stratified according to their baseline characteristics into following four groups: (1) normoglycemia and normal weight group, (2) normoglycemia and overweight/ obese group, (3) prediabetes and normal weight 
Table 1 Baseline characteristics of study participants

\begin{tabular}{|c|c|}
\hline Variable & Result \\
\hline Male & $165(46.9)$ \\
\hline Age (years) & $35.2 \pm 5.3$ \\
\hline Employed & $197(56.0)$ \\
\hline Education, graduation and beyond & $219(62.2)$ \\
\hline \multicolumn{2}{|l|}{ Income per month ${ }^{\mathrm{a}}$} \\
\hline$<20,000 \mathrm{INR}$ & $115(32.8)$ \\
\hline 20,000-50,000 INR & $166(47.3)$ \\
\hline$>50,000 \mathrm{INR}$ & $70(19.9)$ \\
\hline Joint family & $170(48.3)$ \\
\hline Family history of diabetes & $130(36.9)$ \\
\hline Past history of $\mathrm{GDM}^{\mathrm{b}}$ & $109(59.2)$ \\
\hline Postpartum duration (months) ${ }^{c}$ & $43.3 \pm 19.2$ \\
\hline Fasting plasma glucose $(\mathrm{mmol} / \mathrm{L})$ & $5.1 \pm 0.6$ \\
\hline 30-min plasma glucose $(\mathrm{mmol} / \mathrm{L})$ & $8.4 \pm 1.8$ \\
\hline 2-h plasma glucose $(\mathrm{mmol} / \mathrm{L})$ & $6.3 \pm 1.6$ \\
\hline HbAlc (\%) & $5.5 \pm 0.4$ \\
\hline $\mathrm{HbAlc}(\mathrm{mmol} / \mathrm{mol})$ & $36.3 \pm 4.6$ \\
\hline \multicolumn{2}{|l|}{ Glycemic category } \\
\hline Normoglycemia & $244(69.3)$ \\
\hline Prediabetes & $108(30.7)$ \\
\hline $\operatorname{BMI}\left(\mathrm{kg} / \mathrm{m}^{2}\right)$ & $26.0 \pm 4.2$ \\
\hline Normal weight & $158(44.9)$ \\
\hline Overweight & $140(39.8)$ \\
\hline Obesity & $54(15.3)$ \\
\hline WC $(\mathrm{cm})$ & $92.2 \pm 10.3$ \\
\hline WC, women $(\mathrm{cm})$ & $90.8 \pm 11.7$ \\
\hline WC, men $(\mathrm{cm})$ & $93.8 \pm 8.2$ \\
\hline Elevated WC & $276(78.4)$ \\
\hline Elevated WC, women & $158(84.5)$ \\
\hline Elevated WC, men & $118(71.5)$ \\
\hline $\operatorname{SBP}(\mathrm{mmHg})^{\mathrm{a}}$ & $113.0 \pm 15.2$ \\
\hline $\mathrm{DBP}(\mathrm{mmHg})^{\mathrm{a}}$ & $74.9 \pm 10.4$ \\
\hline
\end{tabular}

Table 1 continued

\begin{tabular}{ll}
\hline Variable & Result \\
\hline Hypertension $^{\mathrm{a}}$ & $34(9.7)$ \\
\hline
\end{tabular}

Data expressed as $n, \%$, mean \pm SD or median (q25-q75), as appropriate

$B M I$ body mass index, $D B P$ diastolic blood pressure, GDM gestational diabetes mellitus, INR Indian rupees, $S B P$ systolic blood pressure, $W C$ waist circumference

a $n=351$

b $n=184$

c $n=188$

group, (4) prediabetes and overweight/obese group (Figs. 1 and 2).

\section{Progression in Glycemic Variables and Changes in Insulin Indices in Between Two Time Points}

The mean duration between two study evaluations was $21.5 \pm 7.1$ months [non-infected group, $21.9 \pm 8.5$ months; infected group, $20.9 \pm 5.0$ months]. A progression in glycemic and BMI categories was evident in $71(20.2 \%)$ and $56(15.9 \%)$ participants, respectively. In the glycemic category, progression from normoglycemia to prediabetes occurred in 53 (15.1\%), from prediabetes to diabetes in $16(4.6 \%)$, and from normoglycemia to diabetes in $2(0.6 \%)$ participants. Similarly, in the BMI category, progression from normal to overweight category occurred in $41(11.7 \%)$, and from overweight to obese category in 15 (4.3\%) participants (Table 2).

Progression in BMI category [34 (21.4\%) vs. $22(11.4 \%), p=0.011]$ was seen in a significantly higher proportion of the participants in the infected group compared to the non-infected group. Similarly, weight increase by $5 \%$ or more was evident in a significantly higher proportion of infected compared to non-infected participants [54 (34.0\%) vs. 46 (23.8\%); $p=0.036]$. Despite the BMI change, progression in glycemic $[33(20.8 \%)$ vs. 38 (19.7\%), $p=0.804]$ category did not differ significantly between the two groups. In the infected group, progression in BMI category occurred in 25 


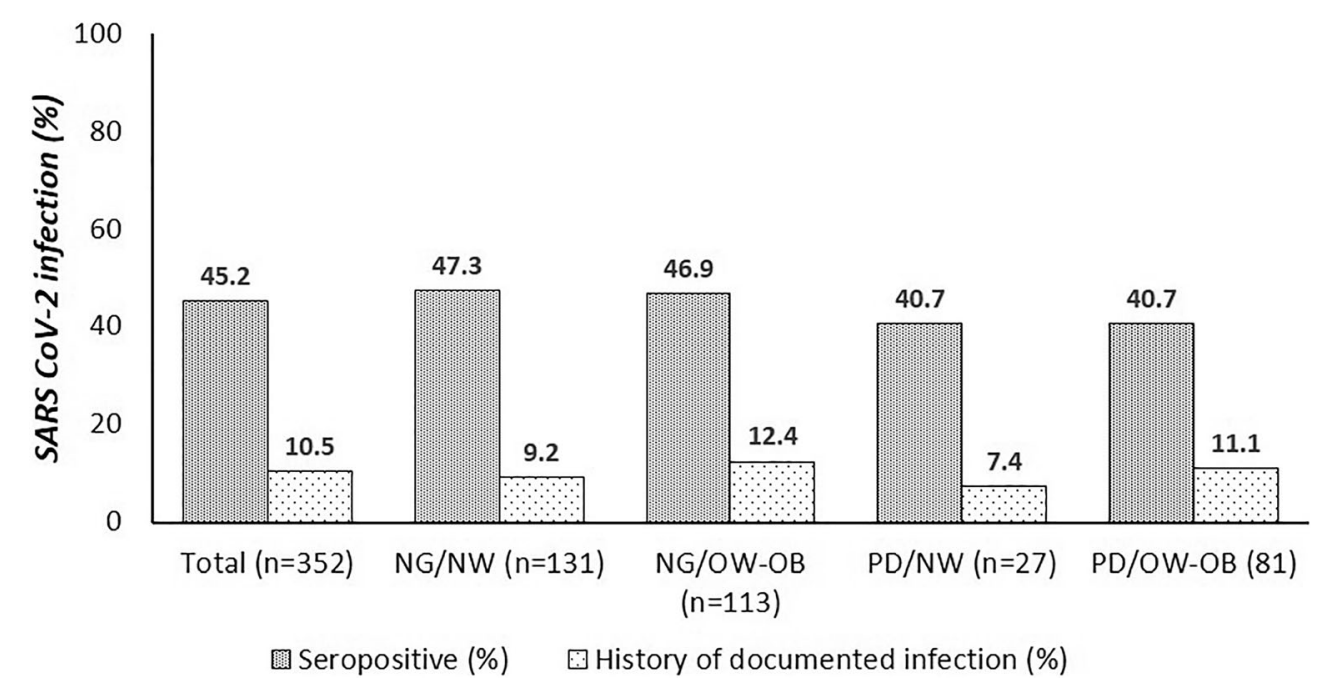

Fig. 1 Bar graph showing prevalence of SARS-CoV-2 seropositivity and documented infection in the study population, stratified according to baseline glycemia and

body weight ( $p=0.744$ and 0.805 , respectively). NG, normoglycemia; NW, normal weight; OW/OB, overweight/obese; PD, prediabetes

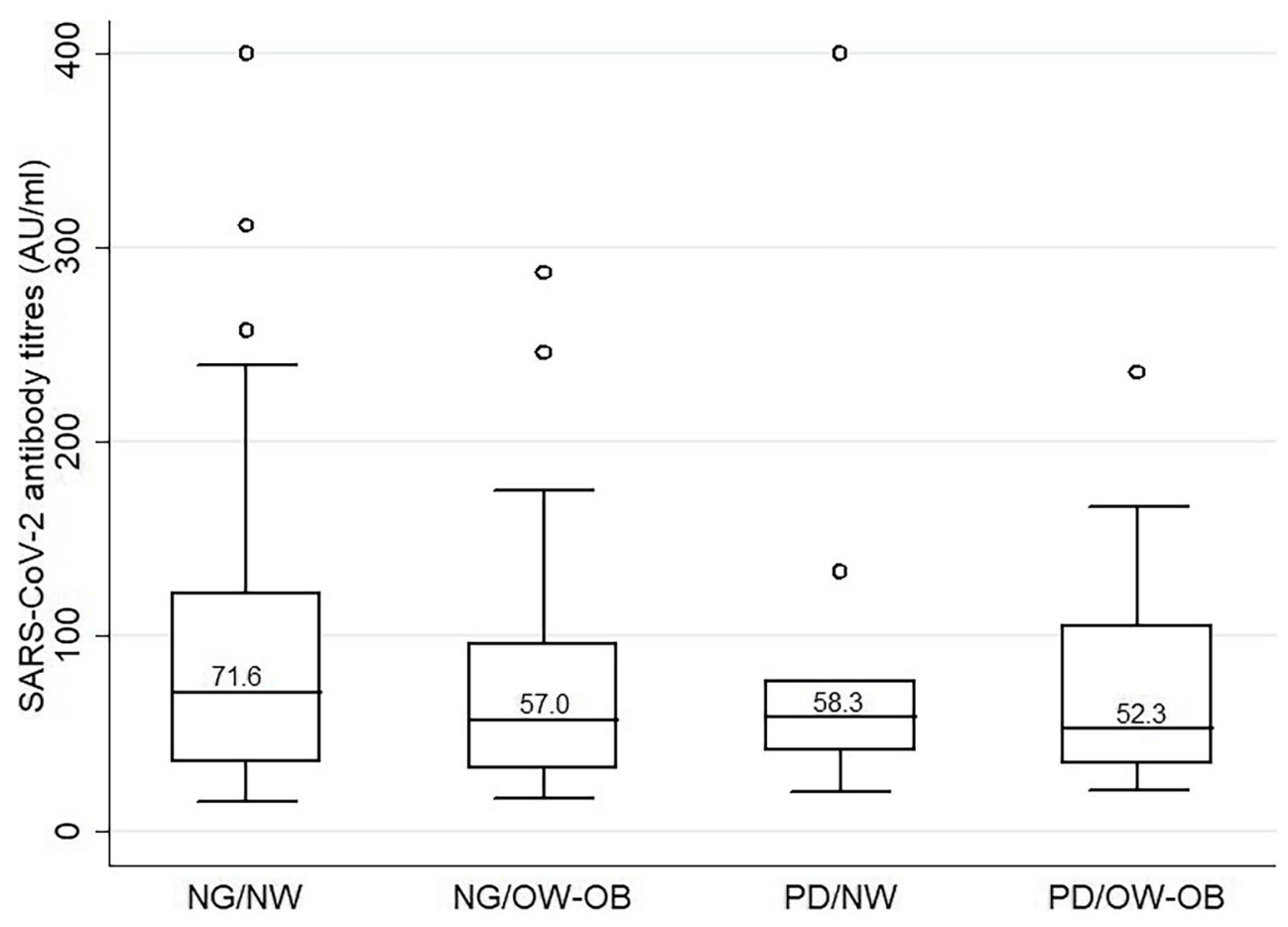

Fig. 2 Box and whisker plot representation of SARSCoV-2 IgG antibody titers in the seropositive participants, stratified according to baseline glycemia and body weight

$(p=0.839) . \mathrm{NG}$, normoglycemia; NW, normal weight; OW/OB, overweight/obese; $\mathrm{PD}$, prediabetes 
Table 2 Progression in glycemic and metabolic variables and changes in insulin indices in SARS-CoV-2 infected and noninfected groups between two study time points

\begin{tabular}{|c|c|c|c|c|}
\hline Variable & Overall $(n=312)$ & $\begin{array}{l}\text { Non-infected } \\
(n=193)\end{array}$ & Infected $(n=159)$ & $p$ value \\
\hline Progression in glycemic category & $71(20.2)$ & $38(19.7)$ & $33(20.8)$ & 0.804 \\
\hline NG to PD & $53(15.1)$ & $29(15.0)$ & $24(15.1)$ & \\
\hline $\mathrm{PD}$ to $\mathrm{DM}$ & $16(4.6)$ & $8(4.2)$ & $8(5.0)$ & 0.965 \\
\hline NG to DM & $2(0.6)$ & $1(0.5)$ & $1(0.6)$ & \\
\hline Progression in BMI category & $56(15.9)$ & $22(11.4)$ & $34(21.4)$ & 0.011 \\
\hline NW to OW & $41(11.7)$ & $15(7.8)$ & $26(16.4)$ & \\
\hline OW to $\mathrm{OB}$ & $15(4.3)$ & $7(3.6)$ & $8(5.0)$ & 0.031 \\
\hline Progression in HOMA-IR category & $113(32.9)$ & $60(31.6)$ & $53(34.4)$ & 0.578 \\
\hline Baseline HOMA-IR & $2.35(1.57,3.58)$ & $2.48(1.75,3.77)$ & $2.30(1.50,3.15)$ & \\
\hline Follow-up HOMA-IR & $2.85(1.92,4.41)$ & $3.05(2.00,4.52)$ & $2.70(1.86,4.14)$ & \\
\hline Delta HOMA-IR ${ }^{a}$ & $0.48(-0.27,1.24)$ & $0.46(-0.40,1.37)$ & $0.49(-0.12,1.16)$ & 0.854 \\
\hline $\begin{array}{l}\text { Progression in Matsuda index } \\
\text { category }\end{array}$ & $95(30.7)$ & $53(30.6)$ & $42(30.9)$ & 0.963 \\
\hline Baseline Matsuda index & $3.72(2.46,5.60)$ & $3.48(2.44,5.33)$ & $4.00(2.52,5.80)$ & \\
\hline Follow-up Matsuda index & $2.91(1.89,4.46)$ & $2.79(1.71,4.40)$ & $3.20(2.14,4.71)$ & \\
\hline Delta Matsuda index ${ }^{\mathrm{b}}$ & $-0.64(-1.85,0.17)$ & $-0.65(-1.88,0.29)$ & $-0.60(-1.75,0.15)$ & 0.833 \\
\hline Progression in oDI category & $66(21.2)$ & $33(18.9)$ & $33(24.1)$ & 0.262 \\
\hline Baseline oDI & $2.31(1.38,3.90)$ & $2.12(1.20,3.19)$ & $2.66(1.72,4.81)$ & \\
\hline Follow-up oDI & $2.40(1.47,4.11)$ & $2.24(1.38,3.86)$ & $2.62(1.72,4.58)$ & \\
\hline Delta oDI ${ }^{c}$ & $-0.07(-0.82,1.26)$ & $0.01(-0.63,1.19)$ & $-0.18(-1.06,1.57)$ & 0.409 \\
\hline
\end{tabular}

Delta values presented for HOMA-IR, Matsuda index, and oDI represent median (IQR) of difference between follow-up and baseline result for individual participants, and should not be seen as difference of median values of these indices provided in the table

$D M$ diabetes mellitus, $N G$ normoglycemia, $o D I$ oral disposition index, $O B$ obese, $O W$ overweight, $P D$ prediabetes

a $n=344$

b $n=309$

c $n=312$

$(20.5 \%)$ and $9(24.3 \%)$ participants with asymptomatic and symptomatic infection, respectively $(p=0.618)$. Similarly, progression in glycemic category occurred in 27 (22.1\%) and $6(16.2 \%)$ participants with asymptomatic and symptomatic infection, respectively $(p=0.437)$. Progression in glycemic category occurred in
$29.4 \%$ and $31.8 \%$ of those with BMI category progression in the infected and non-infected groups, respectively.

The median overall change in HOMA-IR, Matsuda index, and oral disposition index was $0.48(-0.27,1.24),-0.64(-1.85,0.17)$ and $-0.07(-0.82,1.26)$, respectively. There was no 
significant difference in worsening in each of the three insulin indices categories between the infected and non-infected groups [HOMA-IR category: $53(34.4 \%)$ vs. $60(31.6 \%), p=0.578$; Matsuda index category: $42(30.9 \%)$ vs. 53 (30.6\%), $p=0.963$; oDI category: 33 (24.1\%) vs. $33(18.9 \%), p=0.262)$ ] (Table 2$)$.

\section{Change in Insulin Indices in Glycemic Progressors vs. Non-progressors}

The magnitude of change in HOMA-IR over the course of two study visits was significantly higher among participants with glycemic category progression, compared to those without [median (IQR) $1.37(0.24,2.23)$ vs. $0.37(-0.34$, $0.98) ; p<0.001]$. Similarly, the magnitude of change in Matsuda index [median (IQR) -0.99 $(-2.01,-0.31) \quad$ vs. $-0.54 \quad(-1.58,0.41)$; $p=0.005]$ and oDI median (IQR): -0.42 $(-1.07,0.18)$ vs. $0.13(-0.69,1.52) ; p=0.002]$ was significantly higher among participants with glycemic category progression.

\section{Association Between SARS-CoV-2 Infection and Progression of Glycemic and Cardiometabolic Variables}

On logistic regression analysis, the association between SARS-CoV-2 infection and BMI category progression was statistically significant [unadjusted OR $2.11 \quad(95 \% \mathrm{CI}, \quad 1.18-3.79$; $p=0.012)$; fully adjusted OR 2.14 (95\% CI, $1.18-3.90 ; p=0.013)]$. The unadjusted and adjusted ORs for association between SARSCoV-2 infection and progression in glycemic category were all greater than 1.0; however, none was statistically significant. In the fully adjusted model, the OR for glycemic category progression was 1.18 (95\% CI, 0.69-2.01, $p=0.555)$ (Table 3).

\section{DISCUSSION}

A large body of evidence suggesting worse COVID-19 outcomes among patients with diabetes, especially in those with uncontrolled hyperglycemia, is now available [2-4]. However, the data on the other side of the bidirectional relationship, i.e., the magnitude and pathophysiology of SARS-CoV-2-induced glycemic dysfunction, especially in individuals with mild/asymptomatic infection are very scarce. $\mathrm{Wu}$ et al. [24], recently reported that SARS-CoV2 infects beta cells and induces beta cell apoptosis and loss of insulin secretion, in vitro. Similarly, Tang et al. [25] found that the viral antigen is expressed in pancreatic beta cells, and upon infection, the expression of insulin is reduced, while that of glucagon and trypsin 1 is upregulated, indicating beta cell transdifferentiation. However, these data were mainly

Table 3 Unadjusted and adjusted odds ratios (95\% confidence intervals) for the association between SARS-CoV-2 infection and progression of glycemic and metabolic variables

\begin{tabular}{llllll}
\hline Variable (outcome) & Unadjusted OR & Model 1 & Model 2 & Model 3 & Model 4 \\
\hline Glycemic progression & $1.07(0.63,1.80)$ & $1.12(0.66,1.90)$ & $1.08(0.64,1.83)$ & $1.11(0.65,1.88)$ & $1.18(0.69,2.01)$ \\
$p$ value & 0.804 & 0.678 & 0.771 & 0.699 & 0.555 \\
BMI category progression & $2.11(1.18,3.79)$ & $2.05(1.14,3.70)$ & $2.13(1.18,3.82)$ & $2.18(1.21,3.92)$ & $2.14(1.18,3.90)$ \\
$p$ value & 0.012 & 0.017 & 0.012 & 0.010 & 0.013 \\
\hline
\end{tabular}

SARS-CoV-2 severe acute respiratory syndrome coronavirus 2, BMI body mass index

Model 1: Adjusted for age, gender, employment status, and education

Model 2: Adjusted for baseline BMI, and family history of diabetes

Model 3: Adjusted for duration between two study evaluations (years)

Model 4: Model $1+2+3$ 
derived from autopsy studies of patients who died of severe COVID-19. Similarly, clinical observations of an increase in magnitude and severity of hyperglycemia in COVID-19 are based on hospitalized patients [5-7], who invariably suffer from moderate-severe disease. However, in the real world, the number of patients with mild/asymptomatic disease far outnumbers those with moderate/severe disease [26]. Our study evaluated the impact of predominant mild/asymptomatic SARS-CoV-2 infection on worsening of beta cell function and insulin indices and progression of glycemic and cardiometabolic variables, and thus provides a more realistic estimate of the problem at hand.

We found that a significantly higher proportion of participants in the infected group progressed in BMI category, i.e., from normal weight to overweight or from overweight to obesity category. Besides, even after adjustment for multiple covariates, the association between SARS-CoV-2 infection and BMI category progression remained significant (the adjusted odds for progression were nearly twofold higher in the infected group compared to the non-infected group). The existing data suggest that patients with COVID-19 are at a high risk of acute weight loss, owing to several factors such as systemic inflammation, loss of appetite, and loss of smell and taste. In a recent study by Di Filippo et al. [27], 213 patients with COVID19 were evaluated at a median duration of 23 days following recovery; median weight loss was $2.3 \mathrm{~kg}$ and nearly $30 \%$ participants lost more than $5 \%$ of their initial (pre-morbid) weight. A majority (73\%) of patients were hospitalized, implying a severe disease at the time of presentation and were evaluated relatively early following their recovery from viral infection, which explains the divergence of this study's findings with ours. However, the association of viral infection with weight gain seen in our study remains intriguing. We hypothesize that the association results either from a direct link between SARS-CoV-2 and adipose tissue or through indirect mechanisms such as reduced physical activity (resulting from long COVID symptoms such as fatigue and myalgia), and erratic dietary and sleep patterns [28].
Conversely, it is possible that participants who gained weight, and thus progressed in BMI category, were more susceptible to develop COVID-19 (than the other way round, i.e., SARS-CoV-2 infection led to weight gain) [29]. Clearly, these data need further validation in larger studies, and with a longer duration of participant follow-up.

Despite the BMI change, progression in glycemic category was not significantly different between the two groups. The unadjusted and adjusted odds ratios for associations between viral infection and glycemic category progression were greater than 1.0; however, these were not statistically significant. In terms of insulin indices, the measures of insulin resistance (HOMA-IR), insulin sensitivity (Matsuda index), and beta cell function (oral disposition index) worsened in the entire cohort over study duration, especially in glycemic category progressors. However, there was no significant difference between the infected and non-infected groups for worsening of any of the three insulin index categories. Thus, our study suggests that unlike moderate-severe COVID-19, mild/asymptomatic disease is not associated with significant deterioration of beta cell function, insulin resistance, and glycemic parameters, at least in the short term. However, if the effects on these parameters are to be mediated through BMI, it is possible that these may only be revealed on a long-term follow-up of this cohort.

The strengths of our study are its novelty, a large sample size, a longitudinal design, and a comprehensive evaluation of glycemic and cardiometabolic variables and insulin indices at both time points. We reported data on patients with mild/asymptomatic infection, which is more reflective of the real-world scenario. We also adjusted for various covariates, including the duration between two study visits, in order to discern whether and to what magnitude SARS-CoV-2 contributes to the progression, beyond the usual risk factors. We acknowledge certain limitations of this study. Our study cohort comprised a selected group of relatively healthy young individuals who were followed up at a single center. We do not have information on the exact time of onset of infection in 
study participants; however, the duration between two study evaluations was similar in the infected and non-infected groups. The duration of follow-up following the index infection was also short (less than 1 year). The virulence of SARS-CoV-2 is known to vary across the globe [30], and so could its impact on various metabolic parameters. Therefore, this work should be supplemented with more studies performed globally, in different patient populations, and at longer periods following the index infection.

\section{CONCLUSION}

This longitudinal study, for the first time, evaluated the impact of SARS-CoV-2 infection on progression of glycemic and cardiometabolic parameters and insulin indices in a South Asian cohort. The presence of viral infection was associated with a significant progression in BMI category, i.e., from normal weight to overweight or from overweight to obese category. The effect of infection on progression of glycemia and insulin indices categories was not evident; however, this remains worthy of further investigation in a long-term follow-up of the same cohort.

\section{ACKNOWLEDGEMENTS}

The author thank study participants for generously donating their time and information.

Funding. No funding or sponsorship was received for this study or publication of this article.

Authorship. All named authors meet the International Committee of Medical Journal Editors (ICMJE) criteria for authorship for this article, take responsibility for the integrity of the work as a whole, and have given their approval for this version to be published.

Authors' Contributions. AG, YG and NT conceptualised this research and were involved in execution, analysis, manuscript preparation and final approval of publication of this work. MK helped with the statistical part and with initial planning, and final analysis of data, preparation of manuscript and final approval of publication of this work. NB helped in providing inputs in planning, patient recruitment, manuscript editing and in final approval of publication of this work. YG is the guarantor of this work and has full access to the data.

Disclosures. Alpesh Goyal, Yashdeep Gupta, Mani Kalaivani, Neerja Bhatla and Nikhil Tandon have nothing to disclose.

Compliance with Ethics Guidelines. The study was approved by the institutional ethics committee (IEC ref. no. IEC-110/05.02.2021) and informed consent was obtained from all participants. The study was conducted in accordance with the International Conference on Harmonisation Guidelines for Good Clinical Practice and the Declaration of Helsinki.

Data Availability. The datasets generated during and/or analysed during the current study are available from the corresponding author on reasonable request.

Open Access. This article is licensed under a Creative Commons Attribution-NonCommercial 4.0 International License, which permits any non-commercial use, sharing, adaptation, distribution and reproduction in any medium or format, as long as you give appropriate credit to the original author(s) and the source, provide a link to the Creative Commons licence, and indicate if changes were made. The images or other third party material in this article are included in the article's Creative Commons licence, unless indicated otherwise in a credit line to the material. If material is not included in the article's Creative Commons licence and your intended use is not permitted by statutory regulation or exceeds the permitted use, you will need to obtain permission directly from the copyright holder. To view a copy of this licence, visit http://creativecommons.org/licenses/bync/4.0/. 


\section{REFERENCES}

1. WHO Coronavirus Disease (COVID-19) Dashboard. 2021. https://covid19.who.int/. Accessed $15 \mathrm{Jul}$ 2021.

2. Wang S, Ma P, Zhang S, et al. Fasting blood glucose at admission is an independent predictor for 28-day mortality in patients with COVID-19 without previous diagnosis of diabetes: a multi-centre retrospective study. Diabetologia. 2020;63(10):2102-11.

3. Zhu L, She Z, Cheng X, et al. Association of blood glucose control and outcomes in patients with COVID-19 and pre-existing type 2 diabetes. Cell Metab. 2020;31:1-10.

4. Singh AK, Gillies CL, Singh R, et al. Prevalence of co-morbidities and their association with mortality in patients with COVID-19: a systematic review and meta-analysis. Diabetes Obes Metab. 2020;22(10): 1915-24.

5. Unsworth R, Wallace S, Oliver NS, et al. New-onset type 1 diabetes in children during COVID-19: multicenter regional findings in the U.K. Diabetes Care. 2020;43(11):e170-1.

6. Armeni E, Aziz U, Qamar S, et al. Protracted ketonaemia in hyperglycaemic emergencies in COVID19: a retrospective case series. Lancet Diabetes Endocrinol. 2020;8(8):660-3. https://doi.org/10. 1016/S2213-8587(20)30221-7.

7. Li J, Wang X, Chen J, Zuo X, Zhang H, Deng A. COVID-19 infection may cause ketosis and ketoacidosis. Diabetes Obes Metab. 2020;22(10): 1935-41.

8. Rubino F, Amiel SA, Zimmet P, et al. New-onset diabetes in Covid-19. N Engl J Med. 2020;383(8): 789-90.

9. Ramachandran A, Ma RC, Snehalatha C. Diabetes in Asia. Lancet. 2010;375(9712):408-18.

10. Staimez LR, Deepa M, Ali MK, Mohan V, Hanson RL, Narayan KMV. Tale of two Indians: heterogeneity in type 2 diabetes pathophysiology. Diabetes Metab Res Rev. 2019;35(8):e3192.

11. Goyal A, Gupta Y, Kalaivani M, et al. Long term ( $>1$ year) postpartum glucose tolerance status among Indian women with history of gestational diabetes mellitus (GDM) diagnosed by IADPSG criteria. Diabetes Res Clin Pract. 2018;142:154-61.

12. Goyal A, Gupta Y, Kalaivani M, et al. Concordance of glycaemic and cardiometabolic traits between Indian women with history of gestational diabetes mellitus and their spouses: an opportunity to target the household. Diabetologia. 2019;62(8):1357-65.

13. Matthews DR, Hosker JP, Rudenski AS, Naylor BA, Treacher DF, Turner RC. Homeostasis model assessment: insulin resistance and beta-cell function from fasting plasma glucose and insulin concentrations in man. Diabetologia. 1985;28(7): 412-9.

14. Phillips DIW, Clark PM, Hales CN, Osmond C. Understanding oral glucose tolerance: comparison of glucose or insulin measurements during the oral glucose tolerance test with specific measurements of insulin resistance and secretion. Diabet Med. 1994;11(3):286-92.

15. Utzschneider KM, Prigeon RL, Faulenbach MV, et al. Oral disposition index predicts the development of future diabetes above and beyond fasting and two hour glucose levels. Diabet Care. 2009;32(2):335-41.

16. EB Calculator for Matsuda Index. 2021. http:// mmatsuda.diabetes-smc.jp/MIndex.html. Accessed 10 Apr 2021.

17. Matsuda M, DeFronzo RA. Insulin sensitivity indices obtained from oral glucose tolerance testing: comparison with the euglycemic insulin clamp. Diabetes Care. 1999;22:1462-70.

18. The Hindu. COVID-19 vaccines for seniors and 45-plus with comorbidities from March. 2021. https://www.thehindu.com/news/national/covid19-vaccination-for-senior-citizens-from-march-1/ article33922897.ece. Accessed 10 Apr 2021.

19. American Diabetes Association. 2. Classification and diagnosis of diabetes: standards of medical care in diabetes-2020. Diabetes Care. 2020;43(Suppl 1): S14-31.

20. WHO. Obesity and overweight. 2021. https://www. who.int/news-room/fact-sheets/detail/obesity-andoverweight. Accessed 10 Apr 2021.

21. Chobanian AV, Bakris GL, Black HR, et al. The seventh report of the Joint National Committee on Prevention, Detection, Evaluation, and Treatment of High Blood Pressure: the JNC 7 report. JAMA. 2003;289(19):2560-72.

22. Bonelli F, Sarasini A, Zierold C, et al. Clinical and analytical performance of an automated serological test that identifies S1/S2-neutralizing IgG in COVID-19 patients semiquantitatively. J Clin Microbiol. 2020;58(9):e01224-e1320.

23. Sharma N, Sharma P, Basu S, et al. The seroprevalence and trends of SARS-CoV-2 in Delhi, India: a repeated population-based seroepidemiological 
study. medRxiv. 2020. https://doi.org/10.1101/ 2020.12.13.20248123.

24. Wu CT, Lidsky PV, Xiao Y, et al. SARS-CoV-2 infects human pancreatic $\beta$ cells and elicits $\beta$ cell impairment. Cell Metab. 2021;33(8):1565-1576.e5.

25. Tang X, Uhl S, Zhang T, et al. SARS-CoV-2 infection induces beta cell transdifferentiation. Cell Metab. 2021;33(8):1577-1591.e7.

26. Petersen E, Koopmans M, Go U, et al. Comparing SARS-CoV-2 with SARS-CoV and influenza pandemics. Lancet Infect Dis. 2020;20(9):e238-44.

27. Di Filippo L, De Lorenzo R, D’Amico M, et al. COVID-19 is associated with clinically significant weight loss and risk of malnutrition, independent of hospitalisation: a post-hoc analysis of a prospective cohort study. Clin Nutr. 2021;40(4): 2420-6.

28. Logue JK, Franko NM, McCulloch DJ, et al. Sequelae in adults at 6 months after COVID-19 infection. JAMA Netw Open. 2021;4(2):e210830.

29. Pradhan A, Olsson PE. Sex differences in severity and mortality from COVID-19: are males more vulnerable? Biol Sex Differ. 2020;11(1):53.

30. Islam MR, Hoque MN, Rahman MS, et al. Genomewide analysis of SARS-CoV-2 virus strains circulating worldwide implicates heterogeneity. Sci Rep. 2020;10(1):14004. 